\title{
Which intelligence for future networks?
}

\author{
D. Gaïti* and G. Pujolle** \\ *Columbia University \\ Center for Telecommunications Research \\ New York, NY 10027-6699, USA \\ E-mail: gaiti@ctr.columbia.edu \\ **University of Versailles \\ Laboratoire PRiSM \\ 45, avenue des Etats-Unis \\ 78035 Versailles Cedex - France \\ E-mail: Guy.Pujolle@prism.uvsq.fr
}

\begin{abstract}
Service providers should be able to satisfy the demand a new services, to improve the quality of service, to reduce the cost of network service operations and maintenance, to control the performance and to adapt the network to the user demands. In other words, it seems essential to investigate the ways we have in mind to perform such tasks. Three ways may be determined:

- Add a new architectural concept, the Intelligent Network, on the network to be controlled.

- Add an intelligent architecture able to provide a control on the network.

- Introduce intelligence in the nodes of the network to control the protocols.

In this paper we describe these three solutions. First, we present the Intelligent Network solution whose aim is to allow the inclusion of additional capabilities to facilitate provisioning of service, independent of the service and network implementation in a multi-vendor environment. Then, we show that the architecture proposed for the Intelligent Network is very similar to what could be an intelligent architecture. Finally, we propose the introduction of intelligent agents coming from Distributed Artificial Intelligence concepts. These agents could be supported by predetermined nodes of the network.
\end{abstract}




\section{INTRODUCTION}

It has been envisaged that network infrastructures will become highly complex as the evolution of telecommunication toward B-ISDN (Broadband Integrated Services Data Network) take place. Growth in new service deployment and equipment provisioning is expected to mean that very high volumes of information will have to be processed under stringent response-time constraints. Today's users are demanding customization from their telecommunication services. To obtain satisfaction, they are obliged to ask for changes in the switching system of the operator. So, a new service becomes available only when all the switching systems are updated. And, each time a new service is requested, some changes have to be done to introduce new capabilities.

It turns out that the main problem in the future is to control and manage the network and adapt the network to user demands.

Three possibilities are available:

- Add a new network, the Intelligent Network, over the network to be controlled.

- Add an intelligent architecture able to provide a control on the network.

- Introduce intelligence in the nodes of the network to control the protocols.

This paper is divided into 3 sections corresponding to each possibility to introduce the intelligence in future networks. Section 1 presents the Intelligent Network solution. In section 2 , we show that the architecture provided for the Intelligent Network is very similar to an intelligent architecture (as TINA and ODP). In the last part, we propose the introduction of intelligent agents coming from Distributed Artificial Intelligent concepts. Finally, in the conclusion we propose a general solution for introducing intelligence in networks.

\section{THE INTELLIGENT NETWORK}

Intelligent Networks (IN) should promote a very rapid introduction of any new service through a flexible network architecture. This flexibility should be reached using standard interfaces. Intelligent Network is an architectural concept for the operation and provision of new services which is characterized by (see recommendation I.312/Q.1201):

- extensive use of information processing techniques;

- efficient use of network resources;

- modularity and reusability of network functions;

- integrated service creations and implementation by means of the modularized reusable network functions;

- flexible allocation of network functions among physical entities;

- standardized communication between network functions via service independent interfaces;

- service subscriber control of some subscriber-specific service attributes;

- service user control of some user-specific service attributes;

- standardized management of service logic.

To provide these capabilities, it is necessary to define IN functional requirements. Two types of requirements have been defined:

- Service requirements corresponding to the customer needs; 
- Network requirements corresponding to the network operator needs.

Five areas have been identified for service and network requirements that are defined in recommendation I.312/Q.1201.

- Service creation: An activity whereby supplementary services are brought into being through specification phase, development phase and verification phase.

- Service management: An activity to support the proper operation of a service and the administration of information relating to the user/customer and/or the network operator.

- Network management: An activity to support the proper operation of an IN-structured network.

- Service processing: consist of basic call and supplementary service processing which are the serial and/or parallel executions of network functions in a co-ordinated way, such that basic and supplementary services are provided to the customers.

- Network interworking: a process through which several networks (IN to In or IN to nonIN) co-operate to provide a service.

These network capabilities in $\mathrm{IN}$-structured network are shown in Figure 1.

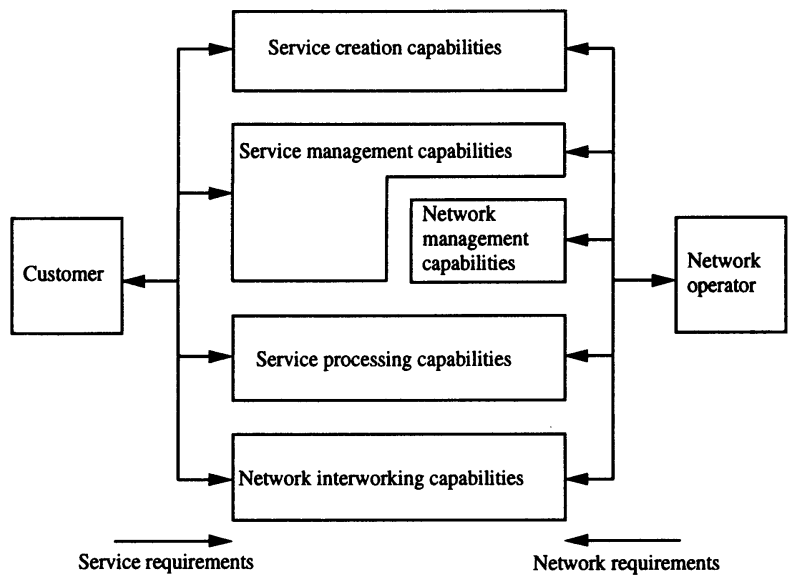

Figure 1. Network capabilities in IN-structured network.

The ITU-T has defined a methodology called INCM. (Intelligent Network Conceptual Model) for specifying the IN architecture. It is not an architecture in itself, but a framework for the design and the description of the $\mathrm{IN}$ architecture [1] [2].

The INCM consists of four planes, where each plane represents a different abstract view of the capabilities provided by an IN-structured network (Figure 2). The planes represent the service view, the global functional view, the distributed functional view and the physical view of IN. 


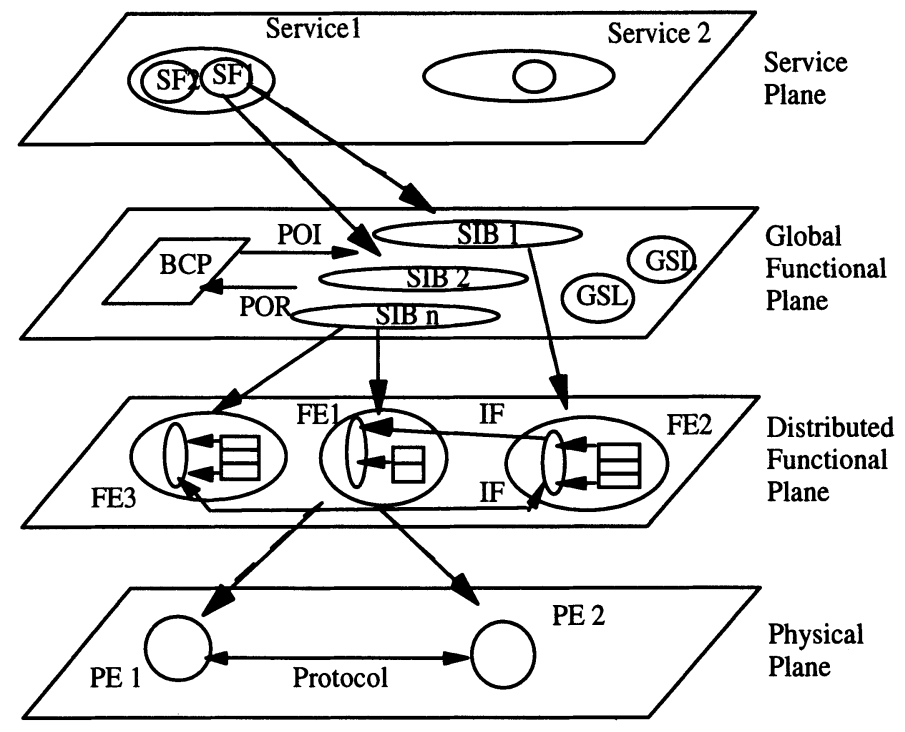

SF: Service Feature

SIB: Service Independent Building block

FE: Functional Entity

PE: Physical Entity
POI: Point Of Initiation

POR: Point Of Return

IF: Information Flows

BCP: Basic Call process

GSL: Global Service Logic

Figure 2. The INCM.

The service plane gives an abstract view to the end-users on the services provided by an INstructured network. A service can be decomposed into one or more Service Features. A Service Feature is built from several Service Independent Building blocks.

The global functional plane defines the Service Independent Building blocks (SIBs) with the following principle: the network is viewed as a single virtual machine. So the distribution is transparent at that level. At this time, 14 SIBs have been defined by ITU-T. This number is insufficient and has to be enlarged by new capabilities. Actually, we have SIBs as "charge", "compare", "distribute", "limit", "log call information", "screen", "status notification", "translate", "user interaction", "verify", "queue", "algorithm", "GSL", "service data management".

In this plane, the Basic Call Process (BCP) and the Global Service Logic (GSL) are defined too.

- The BCP is a specialized SIB that provides the basic call capabilities. SIBs are used to build Global Service Logic (GSL).

- A GSL may be seen as a particular Service Independent Building block and corresponds to a service processing scheme. A GSL is shown in Figure 3. It is presently assumed that the GSL for service processing has no parallelism. The GSL describes the order in which the SIBs can be chained together to accomplish services. 


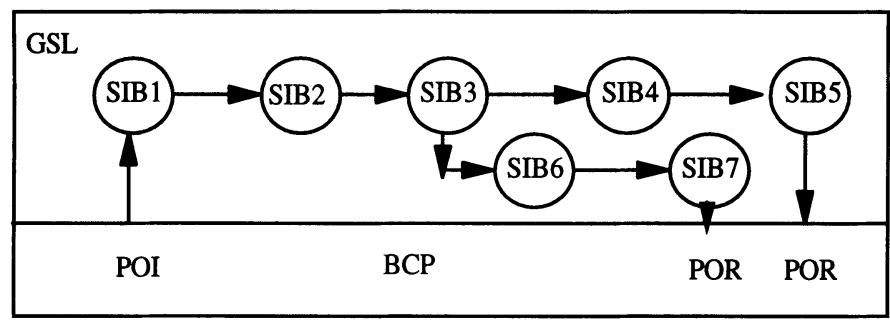

Figure 3. The Global Service Logic.

Two BCP functional interfaces to the GSL have been identified: the Point Of Initiation (POI) and the Point Of Return (POR).

The distributed functional plane models a distributed view of an IN-structured network. This view consists of Functional Entities (FEs). A FE is a unique group of functions in a single location and a subset of the total set of functions required to provide the Service Feature performed by the SIB. A FE is built with Elementary Functions (EFs) and a Functional Entity Action (FEA) as described in Figure 4.

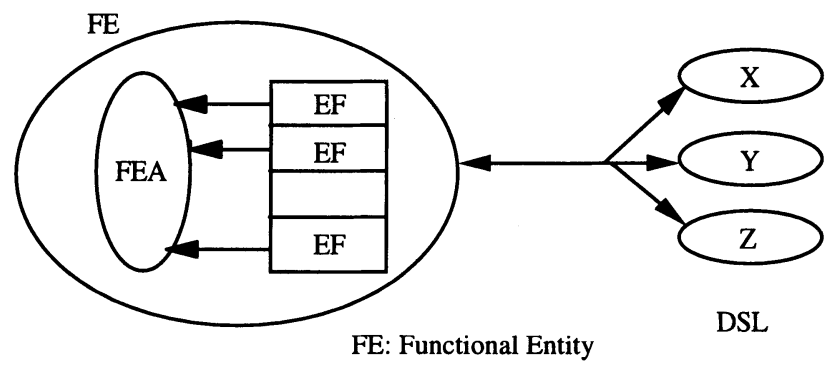

EF: Elementary Function

FEA: Functional Entity Action

DSL: Distributed ServiceLogic

Figure 4. A Functional Entity.

A group of centralized or distributed FEs is mapped onto a SIB. Then a SIB is realized by the interaction between corresponding distributed FEs. These interactions are provided by Information Flows (IF), which are short messages. The advantage of this representation is to have reusable FEs, i.e., a FE can be used by several SIBs.

Moreover, concerning the service logic, there is one set of distributed service logic (DSL) per SIB and it uses FEAs and information flows (see Figure 4).

The physical plane identifies the different Physical Entities (PEs). A Physical Entity consists of one or more Functional Entities. One or more FEs may be mapped onto the same PE, but a FE cannot be split between two PEs. This plane takes into account physical considerations, optimization aspects, and protocols. 
The goal of the IN architecture is to significantly reduce the new service introduction interval with the ability to keep the same communication infrastructure. The IN logical organization [3] may be seen as described in Figure 5.

Two kinds of interfaces have been defined:

- a high-level logical programming interface that defines the access through the interface to logical modules named SIBs (Service Independent Building blocks).

- a resource-control interface that allows the IN infrastructure to control the physical resources. These physical resources can be machines from different vendors.

An important aspect of the objectives of an IN architecture is to come up with an evolutionary perspective for defining new capability sets. The reasoning behind the capability sets is that they serve to address incremental sets of functionalities, achievable within reasonable time frames and with measurable impact on the existing network.

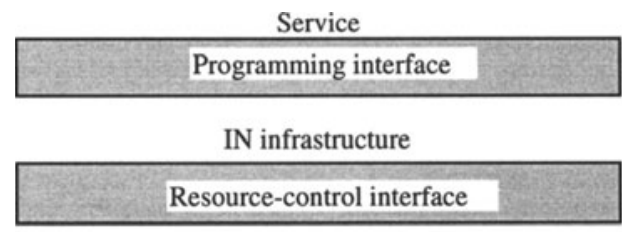

Resources

Figure 5. The logical organization of IN.

A number of significant issues remain to be addressed for introducing a complete intelligence:

- Evolution towards broadband, multi ended, multimedia services. This is expected to include co-operative and high throughput requirements.

- Performance issues. Due to the increase of service traffic, performance objectives need to be placed in an overall IN context.

- Address OAM (Operations And Maintenance) functionalities. The significant impact of OAM issues on network and service operation is critical for the life of future broadbandbased networks.

\section{INTELLIGENT ARCHITECTURE}

In the previous section we described the architecture of the Intelligent Network. Indeed, this architecture is very similar to other architectures able to manage a network. The work to define the $\mathrm{IN}$ architecture was performed in parallel with two other initiatives directed towards the definition of flexible architectures: one coming from ISO and the other one from operators and carriers.

The ISO contribution comes from the ODP (Open Distributed Processing) normalization [4] [5]. The purpose of the ODP standardization is to facilitate systems integration where there is distributed processing and heterogeneity. The conceptual architecture is composed of five viewpoints: 
- enterprise the enterprise viewpoint studies the role of a computer system within an organisation and the interaction between the system and the people within the system

- information the information viewpoint addresses information issues that are influenced by distribution and that affect distribution.

- computational the computational viewpoint models a distributed system as a collection of programs that interacts with each other. The specification of an interaction in this viewpoint includesthe distribution transparencies used in the interaction.

- engineering the engineering viewpoint studies the mechanisms needed for providing different kinds of transparencies.

- technology the technology viewpoint studies how a distributed system can be built using existing technologies including hardware, software and standards.

This architecture is quite identical to the architecture proposed by the TINA (Telecommunication Information Networking Architecture) initiative [6]. This initiative was set up by the major Telecom operator in 1990 to encourage the development of architectural techniques to offer better control in the telecommunication area.

The general TINA Reference Model is shown in Figure 6 [7].

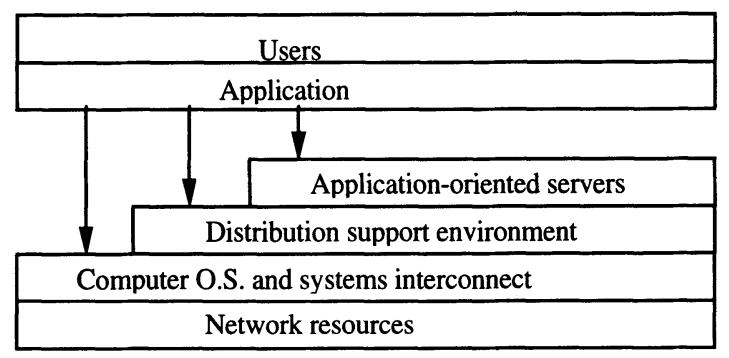

Figure 6. The TINA architecture.

Between the network resources and the applications, the TINA platform offers three layers. The two first ones may be bypassed to access directly the computer operating system.

These three architectures (IN - ODP - TINA) can be compared as follows (Figure 7). They are structured in three main parts:

- An upper part, which is user-oriented. The role of this part is to take into account the users' requirements to provide them with well-suited services.

- A lower part, which is a physical one. It represents the components (hardware and software) on which the system is implemented.

- A medium part, which makes the link between the two others. It realizes the distribution transparencies on one hand and provides an environment for the development of applications on the other hand.

Using the different viewpoints to examine architecture issues encourages a clear separation of concerns, which in turn leads to a better understanding of where to add intelligence:

- Description of the definition of services independently of the way in which that role is automated. 
- Description of the information content of a system independently of the way in which the information is stored or manipulated.

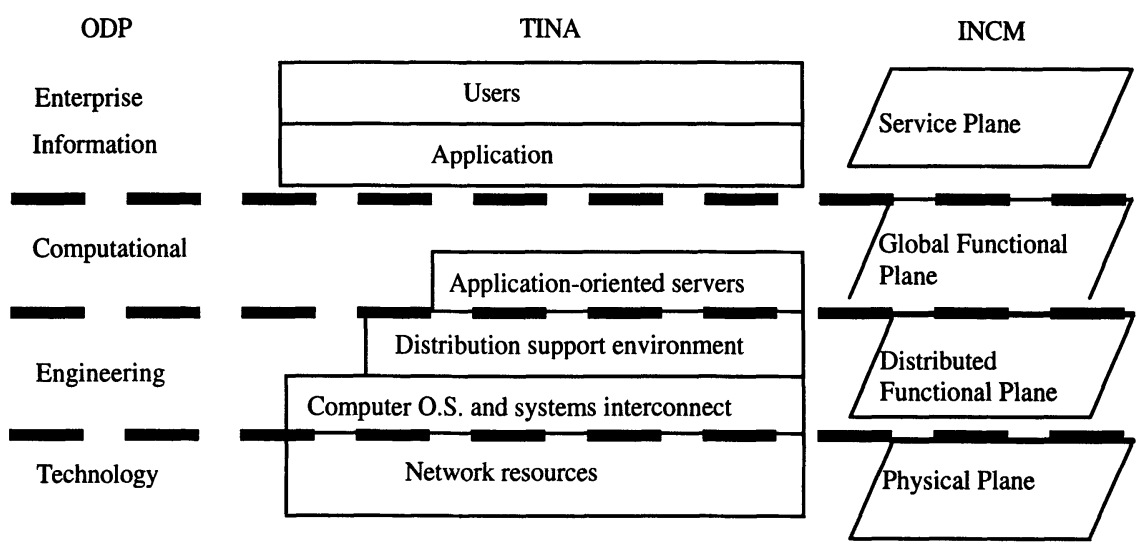

Figure 7. The mapping of the different architectures.

- Description of the application programming environment independently of the way in which that environment is built.

- Description of the components, mechanisms and techniques used to build the system independently of the machines on which they will have to run.

- Description of the system hardware and software independently of the role it plays in the system.

\section{THE DISTRIBUTED ARTIFICIAL INTELLIGENCE APPROACH}

Another way to make the network intelligent is to introduce intelligence in the node through a distributed multi agent system able to control the resources of the network [8]. Distributed Artificial Intelligence concepts give an interesting way to support shared intelligence [9]. This third solution to introduce intelligence in the network can be considered as the only one really "intelligent" in the sense of human intelligence.

The Distributed Artificial Intelligence (DAI) field is often divided into two sub-areas: Distributed Problem Solving (DPS) and multi agent systems. DPS considers how the work of solving a particular problem can be divided among a number of modules that co-operate by dividing and sharing knowledge about the problem and the developing solution [10]. Multiagent systems include intelligent behavior among a collection of possibly pre-existing, autonomous, intelligent "agents", when they can co-ordinate their knowledge, goals, skills, and plans to take action or solve problems [11]. The agents in a multi agent system may be working towards a single global goal or separate individual but interacting goals [12]. Agents in multi agent and DPS systems must share knowledge about problems they face and solutions they reach, but they must also reason about the co-ordination processes among the agents. 
DAI can be useful in domains in which action, perception, and/or control are naturally distributed. It is also a way to reproduce the human behavior when a group of experts work together to realize a task. For these reasons, multi agent systems are envisaged to control future networks.

We propose a methodology to solve any kind of problems that can appear in the control of networks. Our system is generic, even if some specific problems (diagnosis, performance or security management) correspond better to the proposed system. We consider each task of the network control as a distributed problem solving [13].

We propose a global architecture following the principle of a blackboard architecture [14]. This architecture is composed of a collection of agents under the supervision of a control system. The agents co-operate through a knowledge base in writing and reading data corresponding to a given problem. The agent itself has a knowledge part that is controlled to define the problem solving strategy and choose the next action to perform. The agents communicate through an interpreter of messages; they all have a model of the others agents in their environment. The global organization and the agents are described in [15] §3.2.

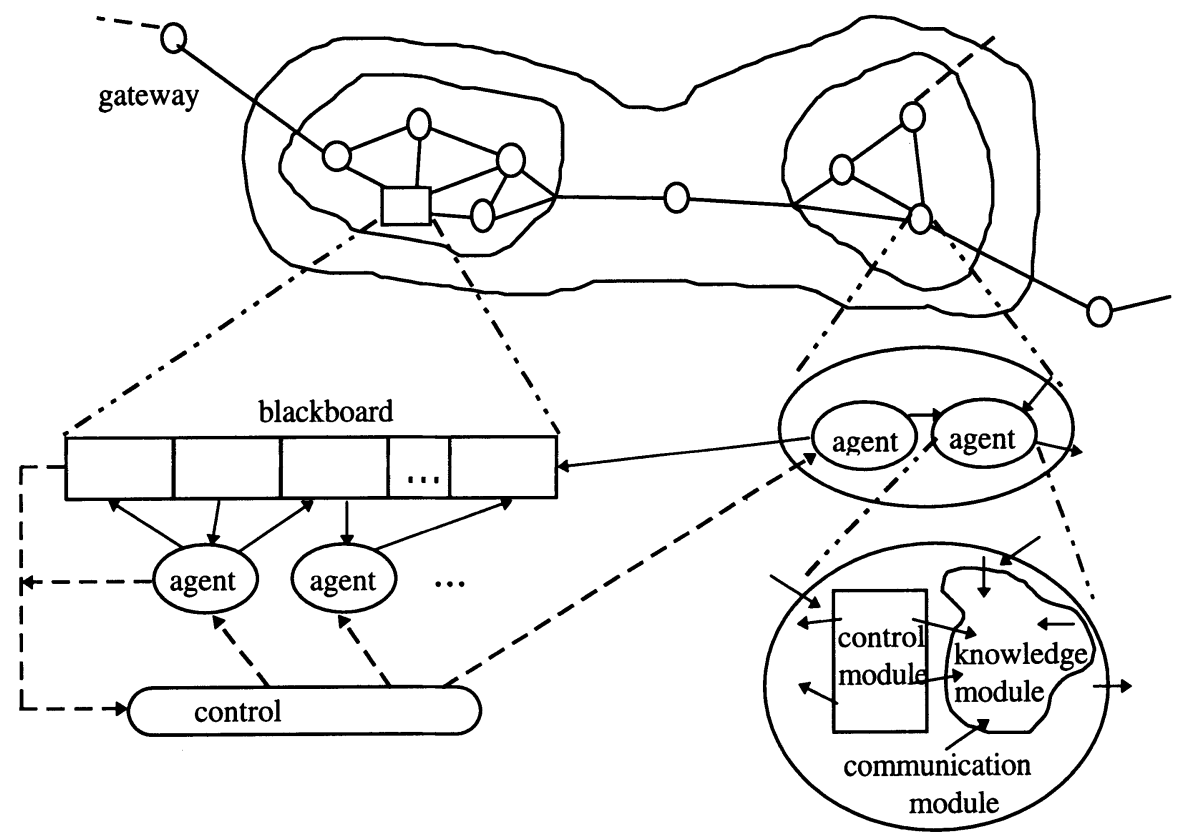

Figure 8. The multi agent system supported by the network.

The integration of this blackboard architecture into the network can be seen as shown Figure 8. One node can have one or several agents depending of the size of the node. We choose one particular node to be responsible for the blackboard.

The blackboard will be more or less used depending on the task we want to achieve. For example, a global management task will take place in the blackboard with the co-ordination and the collaboration of several agents distributed in different nodes. 
But, if we are interested in the control of a node, the agent responsible for this node will solve the task alone with its own knowledge about the task and with its knowledge of the state of the environment. In that case, the agents will be almost totally independent and autonomous. When the network is not too loaded, the agents may communicate each others to update their global view of the state of the network. The blackboard is used in writing to receive information from the nodes through the associated agent when the node is not loaded; this is performed to permit the other agents to update their knowledge of the global state of the system. It is used in reading when an agent of a node wants to update its knowledge. Real time is reached by reducing at the minimum communication requirements. We use this approach for flow control in ATM networks [16] and for an intelligent management in IN [15] [17].

\section{CONCLUSION}

Control and management capabilities play a key role in the viability and success of the emerging broadband networks. Substantial progress has been performed in introducing new technologies in control and management software. An interesting way is to introduce intelligence in operations functions.

We proposed three solutions to introduce some intelligence in the control of future broadband networks. Indeed, these three solutions are based on very different concepts and could be superposed in future networks.

A nice solution would be the introduction of an intelligent architecture (ODP, INCM or TINA) controlled by co-operative agents for problem solving processes. This architecture would be based on concepts developed in $\mathrm{IN}$, distributed networking and distributed artificial intelligence. As a conclusion, we would like to give a sketch of what could be done to introduce simultaneously the three previous types of intelligence.

Let us assume we want to manage and control an ATM network. First, we may adopt an ODP architecture on the global system. On this architecture we implement a distributed model following the concepts introduced in [18]. This architecture is composed of domains that is a first principle for organizing the resources. A domain is a set constituted from resources to be managed in a distributed system and a specific management policy. The second organizing principle concerns the Management Process (MP) that contains two components, the MP view, which is the abstract representation of the resources and of the activities under control and the MP core that executes the management process. It manages and activates the resources through the MP view. At the MP core may be associated the intelligent architecture developed in this paper. Capabilities described in the first part of the paper are provided through the Intelligent Network associated to the ATM network. This architecture may be seen as a platform to support open services.

\section{REFERENCES}

[1] ITU-T, Recommendation I.312/Q.1201, Principles of Intelligent Network Architecture,' October 1992, Geneva. 
[2] José M. Duran and John Visser, International Standards for Intelligent Networks, IEEE Communications Magazine, February 1992.

[3] R. Kung, Rationale for Intelligent Networks, Proc. of the International IFIP Workshop on Open Distributed Processing, Berlin, October 1991.

[4] Basic Reference Model of Open Distributed Processing, Part 2: Descriptive Model ISO 10746-2, 1994.

[5] Basic Reference Model of Open Distributed Processing, Part 3: Prescriptive Model ISO 10746-3, 1994..

[6] T. Boyd, Telecommunication Information Networking Architecture Initiative, Proc. of the International IFIP Workshop on Open Distributed Processing, Berlin, October 1991.

[7] W. Barr, T. Boyd, Y. Inoue, The TINA Initiative, IEEE Communication Magazine, vol. 31, no 3, pp 70-77, march 1993.

[8] I. Rahali and D. Gaïti, A multi-agent system for network management, proceedings of the Second International Symposium on Integrated Network Management, Washington, April 1991.

[9] D. Gaiiti and M.P. Gervais, Artificial Intelligence Environment for Intelligent Network Management, Proc. of the IEEE International Conference on Communication Technology, ICCT'92, pp. 30.05.1-30.05.4, Beijing, China, September 1992.

[10] Alan H. Bond and L. Gasser, eds., Readings in Distributed Artificial Intelligence, San Mateo, Calif.: Morgan Kaufmann, 1988.

[11] E. Werner, The Design of Multi-Agent Systems, Decentralized A.I.-3, Elsevier Science Publishers B.V., 1992.

[12] L. Gasser, Distributed Artificial Intelligence, AI Expert, July 89.

[13] P. Lebouc and P.E. Stern, Distributed Problem Solving in Broadband Telecommunication Network Management, Proc. of the Specialized Conference on Artificial Intelligence, Telecommunications and Computer Systems, 11th International Conference Expert Systems and their Applications, Avignon, 1991.

[14] R. Engelmore et al., Blackboards systems, Reading, Mass.: Addison-Wesley, 1988.

[15] D. Gaïti, An Advanced Management Architecture for IN, International workshop on Intelligent Networks, IFIP, Lappeenranta, Finland, August 1994.

[16] G. Pujolle and D. Gaïti, ATM Flow Control Schemes through a Multi-agent System, Proc. of SICON/ICIE'93, pp. 455-459, IEEE Computer Society Press, Singapour, September 1993.

[17] D. Gaïti, A Proposal for Integrating Intelligent Management in the Intelligent Network Conceptual Model, à paraître dans International Journal on Computer Networks and ISDN Systems, 1994.

[18] D. Gaïti, I2NMA: An Intelligent Integrated Network Management Architecture, International Journal on Network Management, vol 4, 3, 1994. 